MS14-03

\section{Ice-like disorder and phase transitions in cadmium cyanide}

Chloe Coates ${ }^{1}$, Mia Baise ${ }^{2}$, Arkadiy Simonov ${ }^{3}$, Josh Makepeace ${ }^{1}$, Andrew Seel ${ }^{1}$, Ben Slater ${ }^{2}$, Andrew Goodwin ${ }^{1}$

1. Inorganic Chemistry Laboratory, University of Oxford, Oxford, United Kingdom

2. Department of Chemistry, University College London, London, United Kingdom

3. Institute for Crystallography and Material Sciences, University of Freiburg, Freiburg, Germany

\section{email: chloe.coates@chem.ox.ac.uk}

Materials with strongly correlated disorder can be challenging to characterize crystallographically and are thus often poorly understood despite increasing evidence of the link between local deviations from the average structure and advanced material function. $\mathrm{Cd}(\mathrm{CN})_{2}$ has attracted considerable interest for its very strong isotropic negative thermal expansion (NTE). On average, it adopts the interpenetrated diamondoid structure of cubic ice-VII, a topology is able to host a variety of unexpected phenomena, from the two-intwo-out ice rules proton that govern proton disorder in water ice, to magnetic monopoles and loop states in spin-ices. ${ }^{1}$

The correlated disorder in $\mathrm{Cd}(\mathrm{CN})_{2}$ has its signature in the highly structured diffuse scattering, which (unusually) increases in intensity down to a phase transition at $150 \mathrm{~K}$. There exist two types of disorder in $\mathrm{Cd}(\mathrm{CN})_{2} \cdot{ }^{113} \mathrm{Cd} \mathrm{NMR}$ has shown that the $\mathrm{Cd}$ environment favours the two-intwo-out configuration of cyanides which mimics the proton disorder in ice. ${ }^{2}$ This static disorder of the $\mathrm{CN}$ groups is intrinsically related to an off-centering of the $\mathrm{Cd}$ of up to $\sim 1 \AA$ along the $<100>$ directions. ${ }^{3}$

Our interest is in understanding the possible role played by disorder in the NTE of this material. Key indications for the existence of such a link come from comparing the behaviour of $\mathrm{Zn}(\mathrm{CN})_{2}$ and $\mathrm{Cd}(\mathrm{CN})_{2}$ : the former is substantially less disordered, and also shows much weaker NTE. Moreover, previous DFT studies of ordered $\mathrm{Cd}(\mathrm{CN})_{2}$ variants fail to predict accurately its elastic properties, and entirely miss the instability responsible for its $150 \mathrm{~K}$ phase transition.

Using a combination of X-ray and neutron powder, single-crystal and total scattering diffraction data, alongside DFT calculations and ${ }^{113} \mathrm{Cd} \mathrm{NMR}$; I will explore the icerules disorder in $\mathrm{Cd}(\mathrm{CN})_{2}$ and its implications for the microscopic mechanism of NTE. In addition I will present the low temperature structure of $\mathrm{Cd}(\mathrm{CN})_{2}$ and characterise the phase transition in terms of the soft mode responsible for the NTE. Finally, we show that $\mathrm{Cd}(\mathrm{CN})_{2}$ can be considered a dipolar equivalent of a defective spin-ice state capable of hosting monopolar excitations.

References:

[1] S.T. Bramwell et al., (2009) Nature 461, 7266

[2] S-I Nishikiori et al., (1990) Can J Chem 68, 2770

[3] V. E. Fairbank et al., (2012) Phys Rev B, 86, 104113

\section{MS14-04}

\section{Al/Si ordering in mullite}

Paul Benjamin Klar ${ }^{1}$, Iñigo Etxebarria ${ }^{2}$, Gotzon Madariaga ${ }^{1}$

1. Dpto de Física de la Materia Condensada, Universidad del País Vasco UPV/EHU, Bilbao, Spain

2. Dpto de Física Aplicada II, Universidad del País Vasco UPV/EHU, Bilbao, Spain

email: paulbenjamin.klar@ehu.eus

The average crystal structure of mullite $\left(\mathrm{Al}_{4+2 x} \mathrm{Si}_{2-2 x} \mathrm{O}_{10-x}\right)$ is not easy to interpret in terms of the distribution of vacancies and $\mathrm{Al} / \mathrm{Si}$ on the tetrahedral sites. Satellite reflections and diffuse scattering must be investigated to understand the ordering phenomena in the structure. From the reflection conditions of satellite reflections and the corresponding superspace group, the ideal distribution pattern of vacancies and tetrahedra can be directly derived. However, little is known about the distribution of $\mathrm{Si}$ within these tetrahedra, especially with respect to surrounding vacancies. Different NMR and neutron diffraction studies could not give a clear answer [1]. We apply density function theory (DFT) to investigate the $\mathrm{Al} / \mathrm{Si}$ ordering in mullite. As DFT is not feasible with large structures, incommensurately modulated structures are not suitable and commensurate cases or approximations must be used. For this study, commensurate cases with up to 346 atoms in the composition range $0.2 \leq x \leq 0.5$ were generated from a superspace model with full vacancy ordering [2]. For each composition, symmetry compliant permutations of $\mathrm{Al} / \mathrm{Si}$ distributions were analyzed with force-field methods (GULP package [3]). The energetically most stable permutation of each composition was then determined with DFT calculations (VASP) and integrated into the initial superspace model for comparison. The Al/Si ordering for $x>1 / 3$ is defined by one unified superspace model (see Figure below) with Si present in triclusters. A second unified model without $\mathrm{Si}$ in triclusters for $x<1 / 3$ requires the rotation of certain $\mathrm{AlSiO}_{7}$ diclusters with respect to the first model. $\mathrm{Si}_{2} \mathrm{O}_{7}$ diclusters are always sandwiched between two vacancies for the analyzed solid solution range. Structure model refinements in $(3+1) d$ superspace based on synchrotron single crystal X-ray diffraction measurements of mullite with $x \approx 0.4$ cannot distinguish between $\mathrm{Al}$ and $\mathrm{Si}$, but the $\mathrm{Al} / \mathrm{Si}$ ordering can be estimated from the modulated volumes of the tetrahedra. The refined models, which is not fully ordered as only $1^{\text {st }}$ order harmonic functions are used to describe the modulations, agree qualitatively with the predictions from the DFT calculations, i.e. larger volumes are observed where $\mathrm{Al}$ is predicted, and smaller volumes where $\mathrm{Si}$ is predicted. The unified superspace models derived from DFT are considered to represent the ideal, most ordered state of mullite, from which the not fully ordered real structure can be derived. Financial support by FEDER (No. MAT2015-66441-P) and the Basque Government (project No. IT-779-13, PhD grant) is highly appreciated. 